\section{Resistance to colistin in Klebsiella pneumoniae: a 4.0 strain?}

Guido Granata, Nicola Petrosillo

Clinical and Research Department, National Institute for Infectious Diseases

"L. Spallanzani" - IRCCS, Rome, Italy

\begin{abstract}
The global rise of multidrug-resistant gram-negative bacteria represents an increasing threat to patient safety. From the first observation of a carbapenem-resistant gramnegative bacteria a global spread of extendedspectrum beta-lactamases and carbapenemases producing Klebsiella pneumoniae has been observed. Treatment options for multidrug-resistant $K$. pneumoniae are actually limited to combination therapy with some aminoglycosides, tigecycline and to older antimicrobial agents. Unfortunately, the prevalence of colistin-resistant and tigecycline-resistant $K$. pneumoniae is increasing globally. Infection due to colistin-resistant $K$. pneumoniae represents an independent risk factor for mortality. Resistance to colistin in $K$. pneumoniae may be multifactorial, as it is mediated by chromosomal genes or plasmids. The emergence of transmissible, plasmidmediated colistin resistance is an alarming finding. The absence of new agents effective against resistant Gram-negative pathogens means that enhanced surveillance, compliance with infection prevention procedures, and antimicrobial stewardship programs will be required to limit the spread of colistinresistant $K$. pneumoniae.
\end{abstract}

\section{The carbapenemases' globalization}

The global rise of multidrug-resistant (MDR) gram-negative bacteria is alarming and represents an increasing threat to healthcare delivery and patient safety in Europe and beyond. ${ }^{1}$ Klebsiella pneumoniae (K. pneumoniae) is a rod-shaped Gramnegative bacterium representing the most significant member of the Klebsiella genus of Enterobacteriaceae. Since the first description of resistance to $\beta$-lactam antibiotics conferred by the production of extended-spectrum-lactamases (ESBLs) in 1983, ${ }^{2}$ the prevalence of ESBL-producing K. pneumoniae has rapidly increased. Indeed, during the past decades carbapenems were the antibiotic class of choice to face infections caused by penicillins, cephalosporins and quinolones resistant strains of $K$. pneumoni- $a e$, with the (inevitable) first description of a carbapenemase - a specific carbapenemhydrolyzing $\beta$-lactamases - in $1993,{ }^{3}$ followed by the first observation of a carbapenem-resistant $K$. pneumoniae strain in $1999 .^{4-6}$ From then on, a global spread of ESBLs and carbapenemases gram-negative bacteria has been observed, primarily due to $K$. pneumoniae. It is now well known that carbapenem resistance can be the result of various mechanisms including the production of a carbapenemase enzyme (metallo$\beta$-lactamases, $K$. pneumoniae carbapenemases, OXA carbapenemases) and the combination of porins defect plus ESBL or AmpC enzyme production. ${ }^{7}$ Although the spread of carbapenemases appears to be quite recent, NDM, KPC, and OXA carbapenemases are now widely distributed and important reservoirs have been identified: the Indian subcontinent for KPC, NDM and OXA-181, the USA, Israel, Greece and Italy for KPC, and Turkey and North Africa for OXA-48. ${ }^{8}$ The reservoirs of these carbapenemase producer act as significant sources for their dissemination worldwide. ${ }^{8}$ As the rapid increase in the prevalence of ESBLs and carbapenem resistant gramnegative bacteria such as MDR K. pneumoniae is stressing the need for treatment alternatives, treatment options actually in the pipeline are limited to combination therapy with some aminoglycosides, tigecycline and to older antimicrobial agents. Polymyxins, e.g. colistin, has been reconsidered as a valid therapeutic option, in particular for critically ill patients. ${ }^{7,9}$

\section{Fighting with a naked gun}

Tigecycline, an extended-spectrum glycylcycline, acts as a protein synthesis inhibitor reversibly binding the ribosome 30S subunit and interfering with accommodation of aminoacyl-tRNA in the A site of the $16 \mathrm{~S}$ rRNA. ${ }^{10}$ In the U.S., an high rate of tigecycline resistance in $K$. pneumoniae has been recently reported in a multi-center, prospective cohort study on 287 hospitalized patients. ${ }^{11}$ In this study, Van Duin et al. found that $46 \%$ of the cases with carbapenem resistant $K$. pneumonia were also tigecycline-intermediate or -resistant. ${ }^{11}$ In literature, among the variables described as associated with tigecycline resistance there are admission from a nursing home setting and previous antibiotic exposure, ${ }^{11}$ including exposure to tigecycline and antibiotics other than tigecycline. ${ }^{11,12}$ In general terms, it seems that increased antimicrobial resistance to tigecycline in K. pneumoniae is not associated with decreased virulence and fit-
Correspondence: Guido Granata, 2nd Infectious Diseases Division, National Institute for Infectious Diseases "L. Spallanzani", Via Portuense 292, 00149 Rome, Italy .

E-mail: guido.granata@inmi.it.

Key words: Klebsiella pneumoniae, antimicrobial resistance, colistin, tigecycline, plasmid mediated resistance.

Contributions: the authors contributed equally.

Conflict of interest: GG declares no potential conflict of interest; NP receive fee as speaker/scientific board member for Pfizer, MSD, Astellas, Zambon, Angelini, Becton \& Dickinson, Gilead.

Received for publication: 24 February 2017. Revision received: 27 March 2017.

Accepted for publication: 11 April 2017.

This work is licensed under a Creative Commons Attribution-NonCommercial 4.0 International License (CC BY-NC 4.0).

(C) Copyright G. Granata and N. Petrosillo, 2017 Licensee PAGEPress, Italy

Infectious Disease Reports 2017; 9:7104

doi:10.4081/idr.2017.7104

ness, and tigecycline resistance in $K$. pneumoniae bacteremia was found associated with higher mortality rate. ${ }^{13}$ The mechanisms underlying tigecycline resistance are not completely understood. Previous studies attributed tigecycline resistance in Enterobacteriaceae to the increased expression of resistance-nodulation-cell division (RND) type efflux pumps, causing the reduction of the intracellular concentration of tigecycline. ${ }^{10,14,15}$ The RamA-AcrAB and $\operatorname{Rar} A-\mathrm{Oqx} \mathrm{AB}$ efflux pumps pathways are involved in tigecycline resistance in $K$. pneumonia. ${ }^{10}$ The upregulation of transcriptional activators (such as RamA, MarA, RarA, and SoxS) can lead to increased expression of the AcrAB or the OqxAB efflux systems, having a key role in the development of tigecycline resistance. Lin et al. found various mutations in the negative regulatory genes $\operatorname{ram} R$ and $o q x R$ determining overexpression of $\operatorname{ram} A$ and $\operatorname{rar} A$, respectively, ${ }^{12}$ while Fang et al. adopted the construction of a lon gene mutant K. pneumoniae to confirm the association of mutations in the lon gene, an efflux-pump regulator, with tigecycline resistance. ${ }^{16}$ Recently, Villa et al. applied a genome sequencing approach to identify genetic mutations associated with resistance to tigecycline in $K$. pneumoniae. $^{15}$ In this study, the authors identify a potential novel resistance mechanism in the mutation of the $r p s J$ gene. Rps $J$ is a gene coding for riboso- 
mal protein S10-30S, reported to mediate tigecycline resistance in $K$. pneumonia by altering the ribosome structure near the tigecycline-binding site, leading to weaker binding of tigecycline to the $16 \mathrm{~S}$ rRNA. ${ }^{15}$ Finally, in 2014 Nielsen et al. observed the in vivo development of another mechanism of tigecycline resistance, the $\mathrm{kpg} A B C$ gene overexpression due to an IS5 insertion element. ${ }^{17}$ Collectively, these results show the emergence of $K$. pneumoniae strains combining both virulence and multidrug resistance features, a matter of future concern..$^{12,13}$

\section{Last resort drugs}

Colistin (polymyxin E) is a cyclic polypeptide bactericidal agent possessing targeted Gram-negative activity. ${ }^{9}$ The polymyxins are surface active amphipathic agents acting in a detergent-like fashion to disrupt the structure of the cell membrane. The electrostatic interaction between the cationic polypeptide antimicrobial and the anionic lipopolysaccharides of the bacteria outer membrane leads to a leakage of cellular contents and, ultimately, bacterial cell lysis and killing. ${ }^{18}$ Another antibacterial mechanism of the polymyxins has been described, consisting in the inhibition of type II NADH-quinone oxidoreductases (NDH-2), vital respiratory enzymes, in the bacterial inner membrane. ${ }^{19,20}$ Colistin had long been kept as a reserve agent because of safety concerns, in particular regard to nephrotoxicity and neurotoxicity. The exact mechanisms of these adverse events are not well understood and despite the use of newer preparations, it is still substantial with neurotoxicity and nephrotoxicity reported in $4-6 \%$ and $14-53 \%$ of patients, respectively. ${ }^{18,21-24}$

\section{Don't be late for school again, boy}

Unfortunately, with the current increase in the use of colistin for the treatment of MDR Gram-negative bacterial infections, the presence of colistin-resistant gram-negative pathogens has been reported worldwide, with a number of reports of outbreaks of Enterobacteriaceae displaying resistance to colistin..$^{25,26}$ On a survey on 344 isolates of KPC-producing Enterobacteriaceae sent to the Centers for Disease Prevention and Control (CDC) for evaluation from January 2007 through October $2009,9 \%$ of the isolates were resistant to colistin. ${ }^{27} \mathrm{~A}$ large study collecting isolates from 2011-2012 from 31 European medical centres demon- strated significant resistance levels in $K$. pneumonia, $^{28}$ and in the EARS-Net report from $2013,8.8 \%$ of the K. pneumoniae isolates were colistin-resistant, and a majority of the isolates were from Greece, Italy, Romania and Hungary; of concern, $32 \%$ of carbapenem-resistant isolates were resistant to polymyxins..$^{29,30}$ The prevalence of colistin-resistant $K$. pneumoniae is increasing globally, with such infections becoming a critical threat to human health. As reported by Capone et al., infections due to colistinresistant $K$. pneumoniae have a higher mortality rate and colistin-resistant $K$. pneumoniae infections represent an independent risk factor for mortality. ${ }^{31}$

\section{A 4.0 resistant pathogen}

It is well know that resistance to polymyxins in K. pneumoniae can be mediated by chromosomal genes (with the most common mutations in $\operatorname{mgr} B$, phoP/phoQ, pmrA, pmrB, pmrC, and crrABC). ${ }^{20,32}$ Resistance commonly occurs through mutations in the regulatory system PhoPQPmrAB, which leads to upregolation of the pbgPE operon and to the addition of 4amino-4-deoxy-1-arabinose to the bacterial lipopolysaccharide (LPS) structure. This modification alters the charge of the bacterial outer membrane, reducing its interaction with colistin. ${ }^{33-35}$ The wide span of mutations includes silent mutations, point mutations, insertions or deletions. Therefore, resistance to colistin may be multifactorial. Of note, many chromosomally encoded mechanisms of resistance remain to be identified, and it is difficult to extrapolate whether some substitutions identified in proteins known to be involved in LPS biosynthesis lead to resistance or not. Also, the levels of expression of the corresponding genes may vary and consequently influence the level of resistance to polymyxins. ${ }^{20}$ More recently, plasmid mediated colistin resistance mechanism have been reported, ${ }^{36,37}$ i.e. mcr- 1 and mcr-2, and interestingly, Cannatelli et al. have identified a new genetic mechanism responsible for the emergence of colistin resistance, demonstrating that IS5-like elements found on several Klebsiella KPC-encoding plasmids can cause insertional inactivation of the mgrB gene. ${ }^{38}$ MgrB exerts a feedback on the PhoQ/PhoP signaling system. ${ }^{34}$ Therefore, it seems that the acquisition of these plasmids could facilitate the emergence of colistin resistance. ${ }^{38}$

The emergence of transmissible, plasmid-mediated colistin resistance is an alarming finding. These mechanism of transmissible resistance may accelerate the progression of multi-drug resistant Enterobacteriaceae to pan-drug resistant Enterobacteriaceae and may ultimately cause a global spread of pan-drug resistance. ${ }^{36,39}$ Interestingly, as recently discussed by Poirel et al., not only the novel finding of these plasmid mediated mechanisms of resistance represent cause of alarm, but it also may represent an opportunity for the adoption of molecular diagnostic techniques in the field of antimicrobial resistence detection. In fact, as the chromosomally encoded mechanisms of polymyxin resistance can be identified by sequencing specific genes, molecular techniques cannot be certainty envisioned in these case, because of their identifycation detects only potential resistance and is still inferior to phenotypic methods of polymixin resistance detection. Conversely, the molecular identyfication of plasmid-borne $m c r-1 / m c r-2$ genes seems reasonable, since identification of the genes may be considered a signature of resistance and the qualitative genetic results may be translated directly into a nonsusceptibility phenotype..$^{20}$ According to Poirel et al., in the near future screening techniques for mcr- $1 /$ mcr- 2 genes detection may be therefore applied to extensive surveillance studies. $^{20}$

\section{Newer version, newer threat!}

The spread of colistin resistance is a serious cause for concern, particularly in countries with high rates of carbapenem resistant Enterobacteriaceae. Management of colistin resistant $K$. pneumoniae infections presents major challenges and the increasing number of resistance mechanisms reported in such a short time period is alarming. The early identification of colistin resistant strains, at least in hospital settings, may contribute to limitating their spread. ${ }^{40}$ Indeed, among the risk factors found associated with the occurrence of colistin resistance there is prior colistin use and duration, that could relate to the in vivo emergence of colistin resistance after its use, but also factors related to hospitalization such as a history of prior surgical interventions and intensive care unit stay. ${ }^{40}$ If inappropriate usage of colistin in the clinical setting should be avoided, as selection pressure may contribute to colistin resistance due to mutations, the absence of new agents effective against resistant Gram-negative pathogens means that enhanced surveillance, compliance with infection prevention procedures, and antimicrobial stewardship programs will be required to limit the selection and patient-to-patient transmission of the threatening 4.0 colistin-resistant $K$. pneumoniae. 


\section{References}

1. European Centre for Disease Prevention and Control. Plasmid-mediated colistin resistance in Enterobacteriaceae. Stockholm: ECDC; 2016.

2. Knothe H, Shah P, Krcmery V, et al. Transferable resistance to cefotaxime, cefoxitin, cefamandole and cefuroxime in clinical isolates of Klebsiella pneumoniae and Serratia marcescens. Infection 1983;11:315-7.

3. Naas T, Nordmann P. Analysis of a carbapenem-hydrolyzing class A $\beta$-lactamase from Enterobacter cloacae and of its LysR-type regulatory protein. Proc Natl Acad Sci USA 1994;91:7693-7.

4. Koh TH, Babini GS, Woodford N, et al. Carbapenem-hydrolysing IMP-1 betalactamase in Klebsiella pneumoniae from Singapore. Lancet 1999;353:2162.

5. Poirel L, Heritier C, Tolun V, Nordmann P. Emergence of oxacillinase-mediated resistance to imipenem in Klebsiella pneumoniae. Antimicrob Agents Chemother 2004;48:15-22.

6. Yan JJ, Ko WC, Wu JJ. Identification of a plasmid encoding SHV-12, TEM-1, and a variant of IMP-2 metallo-betalactamase, IMP-8, from a clinical isolate of Klebsiella pneumoniae. Antimicrob Agents Chemother 2001; 45:2368-71.

7. Livermore DM. Has the era of untreatable infections arrived? J Antimicrob Chemother 2009;64:i29-36.

8. Nordmann P, Poirel L. The difficult-tocontrol spread of carbapenemase producers among Enterobacteriaceae worldwide. Clin Microbiol Infect 2014;20:821-30.

9. van Duin D, Kaye KS, Neuner EA, Bonomo RA. Carbapenem-resistant Enterobacteriaceae: a review of treatment and outcomes. Diagn Microbiol Infect Dis 2013;75:115-20.

10. Ruilian L, Yujia H, Yiheng $Z$ et al. Tigecycline susceptibility and molecular resistance mechanisms among clinical Klebsiella pneumoniae strains isolated during non-tigecycline treatment. Microbial Drug Resistance. March 2017;23:139-46.

11. van Duin D, Cober E, Richter S, et al. Residence in skilled nursing facilities is associated with tigecycline non-susceptibility in carbapenem-resistant Klebsiella pneumoniae. Infect Control Hosp Epidemiol 2015;36:942-8.

12. Lin YT, Huang YW, Huang HH, et al. In vivo evolution of tigecycline-nonsusceptible Klebsiella pneumoniae strains in patients: relationship between virulence and resistance. Int $\mathrm{J}$
Antimicrob Agents 2016;48:485-91.

13. Juan CH, Huang YW, Lin YT, et al. Risk factors, outcomes, and mechanisms of tigecycline-nonsusceptible Klebsiella pneumoniae bacteremia. Antimicrob Agents Chemother 2016;60:7357-63.

14 Sun Y, Cai Y, Liu X, et al. The emergence of clinical resistance to tigecycline Int $\mathrm{J}$ Antimicrob Agents 2013;41:110-6.

15. Villa L, Feudi C, Fortini D et al. Genomics of KPC-producing Klebsiella pneumoniae sequence type 512 clone highlights the role of RamR and ribosomal S10 protein mutations in conferring tigecycline resistance. Antimicrob Agents Chemother 2014;58:1707-12.

16. Fang L, Chen Q, Shi K et al. Step-wise increase in tigecycline resistance in Klebsiella pneumoniae associated with mutations in ramR, lon and rpsJ. PLoS One 2016;11:e0165019.

17. Nielsen LE, Snesrud EC, Onmus-Leone $\mathrm{F}$, et al. IS5 element integration, a novel mechanism for rapid in vivo emergence of tigecycline nonsusceptibility in Klebsiella pneumoniae. Antimicrob Agents Chemother 2014;58:6151-6.

18. Falagas ME, Kasiakou SK. Colistin: the revival of polymyxins for the management of multidrug-resistant Gram-negative bacterial infections. Clin Infect Dis 2005;40:1333-41.

19. Deris ZZ, Akter J, Sivanesan S, et al. A secondary mode of action of polymyxins against Gram-negative bacteria involves the inhibition of NADHquinone oxidoreductase activity. J Antibiot (Tokyo) 2014;67:147-51.

20. Poirel L, Jayol A, Nordmann P. Polymyxins: antibacterial activity, susceptibility testing, and resistance mechanisms encoded by plasmids or chromosomes. Clin Microbiol Rev 2017;30:557-96.

21. Kwon JA, Lee JE, Huh W, et al. Predictors of acute kidney injury associated with intravenous colistin treatment. Int $\mathrm{J}$ Antimicrob Agents 2010;35:473-7.

22 Holloway KP, Rouphael NG, Wells JB, et al. Polymyxin B and doxycycline use in patients with multidrug-resistant Acinetobacter baumannii infections in the intensive care unit. Ann Pharmacother 2006;40:1939-45.

23. Pogue JM, Lee J, Marchaim D et al. Incidence of and risk factors for colistin-associated nephrotoxicity in a large academic health system. Clin Infect Dis 2011;53:879-84.

24. Durante-Mangoni E, Andini R, Signoriello S, et al. Acute kidney injury during colistin therapy: a prospective study in patients with extensively-drug resistant Acinetobacter baumannii infections. Clin Microbiol Infect 2016;22:984-9.

25. Marchaim D, Chopra T, Pogue JM, et al. Outbreak of colistin-resistant, carbapenem-resistant Klebsiella pneumoniae in metropolitan Detroit, Michigan. Antimicrob Agents Chemother 2011;55:593-9.

26. Mezzatesta ML, Gona F, Caio C, et al. Outbreak of KPC-3-producing, and colistin-resistant, Klebsiella pneumoniae infections in two Sicilian hospitals. Clin Microbiol Infect 2011;17:1444-7.

27. Gupta N, Limbago BM, Patel JB, Kallen AJ. Carbapenem-resistant Enterobacteriaceae: epidemiology and prevention. Clin Infect Dis 2011;53:607.

28. Sader HS, Farrell DJ, Castanheira M, et al. Antimicrobial activity of ceftolozane/tazobactam tested against Pseudomonas aeruginosa and Enterobacteriaceae with various resistance patterns isolated in European hospitals (2011-12). J Antimicrob Chemother 2014;69:2713-22.

29. European Centre for Disease Prevention and Control (ECDC). Antimicrobial resistance surveillance in Europe, 2013. Annual report of the European Antimicrobial Resistance Surveillance Network (EARS-Net). Stockholm: ECDC; 2014.

30. Giske CG. Contemporary resistance trends and mechanisms for the old antibiotics colistin, temocillin, fosfomycin, mecillinam and nitrofurantoin. Clin Microbiol Infect 2015;21:899-905.

31. Capone A, Giannella M, Fortini D, et al. High rate of colistin resistance among patients with carbapenem-resistant Klebsiella pneumoniae infection accounts for an excess of mortality. Clin Microbiol Infect 2013;19:E23-30.

32. Pragasam AK, Shankar C, Veeraraghavan B, et al. Molecular mechanisms of colistin resistance in Klebsiella pneumoniae causing bacteremia from India: a first report. Front Microbiol 2017;7:2135.

33. Helander IM, Kato Y, Kilpelainen I, et al. Characterization of lipopolysaccharides of polymyxin-resistant and polymyxin-sensitive Klebsiella pneumoniae O3. Eur J Biochem 1996;23 7:272-8.

34. Cheng HY, Chen YF, Peng HL. Molecular characterization of the PhoPQ-PmrD-PmrAB mediated pathway regulating polymyxin $\mathrm{B}$ resistance in Klebsiella pneumoniae CG43. 
J Biomed Sci 2010;17:60.

35. Falagas ME, Rafailidis PI, Matthaiou DK. Resistance to polymyxins: mechanisms, frequency and treatment options. Drug Resist Updat 2010;13:132-8.

36. Liu YY, Wang Y, Walsh TR, et al. Emergence of plasmid-mediated colistin resistance mechanism MCR-1 in animals and human beings in China: a microbiological and molecular biological study. Lancet Infect Dis 2016;16:161-8.
37. Di Pilato V, Arena F, Tascini C, et al. MCR-1.2, a new mcr variant carried on a transferable plasmid from a colistinresistant KPC carbapenemase-producing Klebsiella pneumoniae strain of sequence type 512. Antimicrob Agents Chemother 2016;60:5612-5.

38. Cannatelli A, D'Andrea M, Giani T, et al. In vivo emergence of colistin resistance in Klebsiella pneumoniae producing KPC-Type carbapenemases mediated by insertional inactivation of the
PhoQ/PhoP mgrB regulator. Antimicrob Agents Chemother 2013;57:5521-6.

39. Lippa AM, Goulian M. Feedback inhibition in the $\mathrm{PhoQ} / \mathrm{PhoP}$ signaling system by a membrane peptide. PLoS Genet 2009;5:e1000788.

40. Matthaiou DK, Michalopoulos A, Rafailidis PI, et al. Risk factors associated with the isolation of colistin-resistant gram-negative bacteria: a matched case-control study. Crit Care Med 2008;36:807-11. 